\title{
Adsorption and passivation of copper by triazoles in neutral aqueous solution
}

\author{
Yu. I. Kuznetsov, ${ }^{1 *}$ M. O. Agafonkina, ${ }^{1}$ H. S. Shikhaliev, ${ }^{2}$ N. P. Andreeva ${ }^{1}$ \\ and A. Yu. Potapov ${ }^{2}$ \\ ${ }^{1}$ A. N. Frumkin Institute of Physical Chemistry and Electrochemistry, Russian Academy of \\ Sciences, Leninskii pr. 31, Moscow, 119071 Russian Federation \\ ${ }^{2}$ Voronezh State University, Universitetskaya pl. 1, Voronezh, 394006 Russian Federation \\ *E-mail: kuznetsov@ipc.rssi.ru
}

\begin{abstract}
The relationship between hydrophobicity of triazoles and their adsorption and passivation ability in relation to copper is analyzed. Adsorption of triazoles on oxidized copper surface is polymolecular. Adsorption of the first monolayer is described by Frumkin equation with the free energy of adsorption in the range of 51-70 kJ/mol. Certain triazoles are able to effectively inhibit anodic dissolution of copper in neutral chloride solutions. The possible spatial arrangement of the triazoles on the surface of oxidized copper is revealed.
\end{abstract}

Keywords: triazole, 3-amino-1,2,4-triazole, triazole derivatives, copper, monolayer, passivity, free energy of adsorption.

Received: March 13, 2014.

doi: $\underline{10.17675 / 2305-6894-2014-3-2-137-148}$

1,2,3-Benzotriazole (BTA) is the most thoroughly studied corrosion inhibitor of copper among azoles [1-5]. Back in the 1960s, studies of inhibiting properties of BTA demonstrated its high efficiency and the ability to form a protective film with a thickness about 0.5 microns as measured reflectance infrared spectroscopy [3, 4]. Studies of copper corrosion inhibition by BTA continue at present [5-9]. It is partly due to the commercial availability of this very weak $\mathrm{NH}$-acid $\left(\mathrm{p} K_{\mathrm{a}} 8.10 \pm 0.17\right)$, its good solubility in aqueous solutions, high stability (melting point $=100^{\circ} \mathrm{C}$, boiling point $=201-204^{\circ} \mathrm{C}$ at $P=$ $15 \mathrm{mmHg}$ ), and ability to form sparingly soluble compounds with cations of many metals (Ag, $\mathrm{Cu}, \mathrm{Zn}, \mathrm{Ni}, \mathrm{Co}, \mathrm{Pb})[5])$.

Not only BTA derivatives but also some other more hydrophilic triazoles that are surfactants attract the attention of researchers for the protection of copper and its alloys. According to [10], 3-amino-1,2,4-triazole (3-AT) is more efficient than BTA in preventing copper pitting in borate buffer solutions containing chlorides or sulfates. Some triazole derivatives are well studied as corrosion inhibitors of copper and mild steel in acidic media [11-14].)

Incorporation of hydrophobic substituents to 3 -AT structure $[5,15]$ has a beneficial effect on the protection of copper in various environments. Furthermore, tolyltriazole, a 
BTA derivative that is more hydrophobic than BTA itself, is also an efficient corrosion inhibitor of copper-aluminum alloys, both in neutral $(1 \mathrm{M} \mathrm{NaCl})$ and in acidic medium (1 M HCl) [16].

The aim of this study is to compare the adsorption behavior of a series of triazoles on copper and the inhibiting effect on anodic dissolution of copper in aqueous solutions containing chloride ions, and also to analyze the effect of the molecular structure of aminotriazoles (AT) on these factors.

\section{Experimental}

Adsorption and electrochemical studies were conducted in a borate buffer with $\mathrm{pH} 7.40$ based on distilled water [17]. 5-Mercaptopentyl-3-amino-[1,2,4]-triazole (MP-3-AT) and 5-pentyl-3-amino-[1,2,4]-triazole (3-pentyl-AT) synthesized at Voronezh State University and industrial chemical compounds: 3-AT, 4-amino-[1,2,4]-triazole (4-AT) and 1-H-1,2,4triazole were used in this study.

The synthesis of 3-pentyl-AT was carried out by the following procedure. A mixture of $23.2 \mathrm{~g}(0.2 \mathrm{~mol})$ of caproic acid, $27.2 \mathrm{~g}(0.2 \mathrm{~mol})$ of aminoguanidine bicarbonate, and $60 \mathrm{ml}$ of toluene was boiled with a Dean-Stark water trap until water separation in the trap ceased. The pentyl-3-AT crystallized after cooling of the reaction mass was filtered off and washed with toluene and hexane. The dried reaction product was crystallized from a minimum amount of methanol. Yield: $12.3 \mathrm{~g}(40 \%)$, m.p. $=117-119^{\circ} \mathrm{C}$.

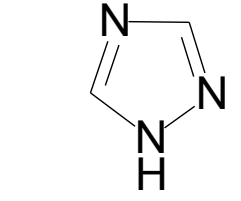

1- $H-1,2,4-$ triazole

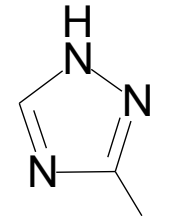

$\mathrm{NH}_{2}$

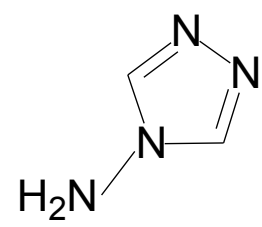

4-amino-[1,2,4]-triazole (4-AT)

3-amino-[1,2,4]-triazole (3-AT)

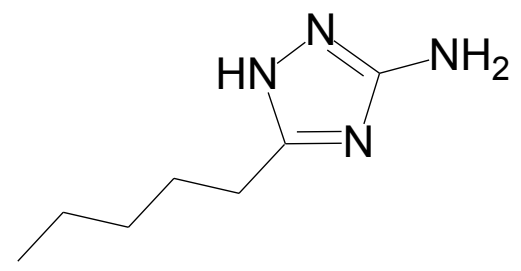

5-pentyl-3-amino-[1,2,4]triazole (3-pentyl-AT )

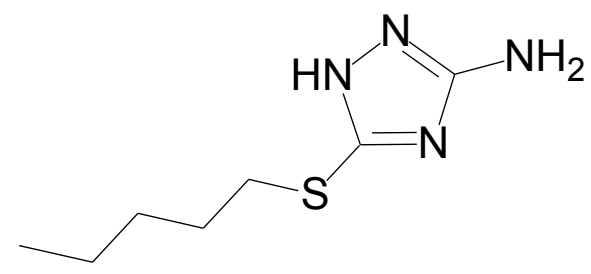

5-mercaptopentyl-3-amino-[1,2,4]-triazole (MP-3-AT)

In the synthesis of MP-3-AT, $26 \mathrm{~g}(0.2 \mathrm{~mol})$ of 5-mercapto-3-amino-[1,2,4]-triazole was dissolved in $80 \mathrm{ml}$ of $10 \%$ aqueous sodium hydroxide solution. $n$-Pentyl iodide ( $35.6 \mathrm{~g}, 0.2 \mathrm{~mol}$ ) was added dropwise over 3 hours to the resulting solution. The precipitate 
of pentylmercapto-5-amino-3-[1,2,4]-triazole hydrochloride was filtered off, washed with water and dried. Yield: $18 \mathrm{~g}(48 \%)$, m.p. $=51-53^{\circ} \mathrm{C}$.

Polarization curves were measured using an IPC-PRO potentiostat on copper electrode (M1) with a surface area of $s=0.75 \mathrm{~cm}^{2}$ in an electrochemical cell with separated electrode spaces. The main electrode was prepared by sanding and degreasing with acetone. The electrode potentials $(E)$ were measured against a silver chloride electrode and then converted to the normal hydrogen scale. A platinum auxiliary electrode was used.

After removal of the air-formed oxide film by cathodic polarization of copper at $E=$ $-0.60 \mathrm{~V}$ for $15 \mathrm{~min}$, the potentiostat was switched off until free corrosion potential $E_{\text {cor }}$ was established. Solutions of $\mathrm{NaCl}(10 \mathrm{mmol} / \mathrm{l})$ and inhibitor were added to the stirred solution (magnetic stirrer). Once a new $E_{\text {cor }}$ value was established, anodic polarization curves were measured with a potential scanning rate of $0.2 \mathrm{mV} / \mathrm{s}$. The pitting potential $\left(E_{\mathrm{pt}}\right)$ was determined by a sharp current increase on the polarization curves with subsequent visual identification of the pitting on the electrode surface. The $E_{\mathrm{pt}}$ measurement error was $0.02 \mathrm{~V}$.

Inhibitor adsorption on passive copper was studied using an RR2000 manual ellipsometer, in a cell designed for simultaneous electrochemical and ellipsometric investigations. A helium-neon laser $\left(\lambda=640 \mathrm{~nm}\right.$, light incident angle $\left.68.5^{\circ}\right)$ was used as a radiation source. The measured ellipsometric angles $\Delta$ and $\Psi$ characterize the state of the electrode surface and change when a potential is applied to the electrode and/or an inhibitor is added to the solution. For very thin films $(\leq 10 \mathrm{~nm})$, we employed the Drude equation for homogeneous and isotropic films that relates the changes in the ellipsometric phase shift angle $\delta \Delta$ with the film thickness $(d)$ :

$$
d=-\alpha \delta \Delta=-\alpha\left(\Delta-\Delta_{0}\right),
$$

where $\alpha$ is a proportionality coefficient; $\Delta$ and $\Delta_{0}$ are the ellipsometric angles measured in the presence and in the absence of the film formed, respectively. A film grows at $\delta \Delta<0$ indicates adsorption, whereas at $\delta \Delta>0$ the film becomes thinner that implies desorption.

When addition of the next portion of the inhibitor no longer changes angle $\Delta$, we assume that surface coverage $(\theta)$ approaches 1.0, i.e., formation of a conditional monolayer is completed. The dependence of the $(-\delta \Delta)$ value on $\lg C_{\text {inh }}$ was converted to the isotherm of inhibitor adsorption. After that, we determined which of the known adsorption equations can be used to describe it and finally estimated the standard free energy of adsorption $\left(-\Delta G_{\mathrm{a}}^{0}\right)$. The following criterion was used to select the equation for describing the experimental isotherms. If the isotherm has an S-like shape and $\theta$ (in the medium surface coverage range) is proportional to $\lg C$, i.e., the experimental data can be described by the Frumkin isotherm:

$$
B C=[\theta /(1-\theta)] \exp (-2 \mathrm{a} \theta)
$$


where $B$ is the adsorption constant related to the free energy of adsorption $\left(-\Delta G_{\mathrm{a}}^{0}\right)$ by the equation $B=\left[\exp \left(-\Delta G_{\mathrm{a}}^{0} / R T\right)\right] / 55.5$, and $a$ is the attraction constant characterizing the interaction between the adsorbate particles.

To find $\ln B$, the resulting isotherm was rectified in the coordinates: $X=\theta, Y=$ $\ln [\theta /(1-\theta) C]$. The intersection of the tangent with the $Y$ axis in the medium coverage range determines the value of $\ln B$. The statistical evaluation of the adequacy of the experimental isotherms to theoretical models was based on the $F$-criterion. It is shown that they can be adequately described by Eq. (2) at $95 \%$ adequacy level.

Measurements of inhibitor adsorption on oxidized copper surface from a borate buffer solution were carried out on electrodes pre-reduced at a cathodic potential $(E=$ $-0.60 \mathrm{~V}, 15 \mathrm{~min}$ ) followed by oxidation at $E=0.0 \mathrm{~V}$. After $40-60 \mathrm{~min}$, the changes in $(-\delta \Delta)$ associated with a growth of $\mathrm{Cu}_{2} \mathrm{O}$ oxide do not exceed $\left(-0.8^{\circ}\right)$ and do not change for $24 \mathrm{~h}$. This constancy of the ellipsometric parameters after electrode oxidation indicates that a stable surface state is reached.

By comparing the changes in $(-\delta \Delta)$ that occur upon addition of a compound to the solution with the initial surface parameters, the adsorption isotherm can be obtained [18]. To do so, a concentrated inhibitor was introduced into the solution and any changes in the ellipsometric angles over time were recorded. To plot ellipsometric parameters against the concentration of the adsorbed reactant $\left(C_{\text {in }}\right)$, the values of $\Delta$ and $\psi$ angles that ceased changing over time were used.

\section{Experimental results and discussion}

Unsubstituted 1-H-1,2,4-triazole is a weak NH-acid and an even weaker base [19]. It is readily soluble in water, i.e. relatively hydrophilic as a chemical compound. Usually the logarithm of the partition coefficient of a compound in a system of two immiscible liquids (octanol-water), $\log P$, is taken as the criterion of hydrophobicity of that compound. In order to allow a correction for the dissociation of the acid, the quantity characterizing the hydrophobicity of anions can be calculated [20]:

$$
\log D=\log P-\log \left[+10\left(\mathrm{pH}-\mathrm{p} K_{\mathrm{a}}\right)\right]
$$

As one can see from Table 1, calculation of these quantities confirms the hydrophilicity of unsubstituted triazole. All adsorption isotherms of triazoles on copper are S-shaped.

Adsorption of unsubstituted 1- $H$-1,2,4-triazole (Fig. 1) starts at rather low $C_{\text {in }}$ values $(\lg C \sim-11)$. This indicates that triazole has a high adsorption capability on copper, probably due to chemisorption. After the formation of a conditional monolayer (plateau on the curve up to the dotted line) is becomes possible to calculate the adsorption isotherm, which is adequately described by Eq. (2) with the values $\left(-\Delta G_{\mathrm{a}}^{0}\right)=64 \mathrm{~kJ} / \mathrm{mol}, a=1.7$ (Table). The high value of $\left(-\Delta G_{\mathrm{a}}^{0}\right)$, which is significantly greater than the corresponding 
value for BTA, one of the best-known inhibitors of this class, with $\left(-\Delta G_{\mathrm{a}}^{0}\right)=50.5 \mathrm{~kJ} / \mathrm{mol}$ (and even for 5-chloroBTA, its more efficient derivative with $\left(-\Delta G_{\mathrm{a}}^{0}\right)=58.5 \mathrm{~kJ} / \mathrm{mol}[21]$ ), is an additional reason for the assumption about the chemisorption of $1-H-1,2,4$-triazole. It is essential that chemisorptive interaction of these benzotriazoles with the surface of copper have been proved by various physical and chemical methods used to study surfaces, for example, XPS [5, 22, 23].

Table 1. Dependence of $\mathrm{p} K_{\mathrm{a}}$, the logarithm of the distribution coefficient $\log P, \log D$ and $\left(-\Delta G_{\mathrm{a}}^{0}\right)$ on the chemical structure of the inhibitor.

\begin{tabular}{lccccc}
\hline & $\begin{array}{c}\text { 4- } \\
\text { aminotriazole }\end{array}$ & $\begin{array}{c}\mathbf{3 -} \\
\text { aminotriazole }\end{array}$ & $\begin{array}{c}\mathbf{1 - H - 1 , 2 , 4 -} \\
\text { triazole }\end{array}$ & $\begin{array}{c}\text { P-3- } \\
\text { aminotriazole }\end{array}$ & $\begin{array}{c}\text { PM-3- } \\
\text { aminotriazole }\end{array}$ \\
\hline $\log P$ & -1.38 & -0.87 & -0.58 & 1.13 & 2.17 \\
\hline $\mathrm{p} K_{\mathrm{a}}($ acid $)$ & $\mathrm{N} / \mathrm{A}$ & 10.5 & 10.0 & 10.18 & 10.18 \\
$\mathrm{p} K_{\mathrm{b}}$ (base) & $3.94 ; 2.63$ & $4.2[24]$ & $2.2[19]$ & & \\
\hline $\log D$ & -1.38 & -1.06 & -0.58 & 1.13 & 2.16 \\
\hline$\left(-\Delta G_{\mathrm{a}}^{0}\right)$, & 51 & 53 & 64 & 69 & 70 \\
$\mathrm{~kJ} / \mathrm{mol}$ & & & & & \\
\hline
\end{tabular}

After the formation of a conditional monolayer, further measurements of angle $\Delta$ do not show permanent values of $(-\delta \Delta)$, as it changes over time. This may be due to the fact that copper cations slowly accumulate in solution and form complexes with the inhibitor. These complexes start adsorbing to form a second layer. For this reason, the formation of the second layer is shown by a dotted line on the isotherm.

Introduction of a hydrophilic amino group into the triazole ring can weaken adsorption because it increases the energy of interaction of the compound with the solvent, i.e., water. Quantitative evaluation of AT hydrophobicity confirms an increase in their hydrophilicity compared with triazole itself (Table 1). It is especially evident in the case of 4-AT, which is also devoid of even weak acidic properties.

For comparison with the absorption properties of 1- $H-1,2,4$-triazole, the adsorption isotherms of 3-AT and 4-AT obtained previously under the same conditions [21] are demonstrated in Fig. 1 . They are also described by Eq. (2) with the parameters $\left(-\Delta G_{\mathrm{a}}^{0}\right)=$ $53 \mathrm{~kJ} / \mathrm{mol}, a=1.3$ and $\left(-\Delta G_{\mathrm{a}}^{0}\right)=51 \mathrm{~kJ} / \mathrm{mol}, a=2.0$, respectively, for 3-AT and 4-AT (Table 1). These isotherms are by two orders to the right along the axis of concentrations than the isotherm for 1-H-1,2,4-triazole, which indicates the role of hydrophobicity (hydrophilicity) in the adsorption properties of a series of compounds. The adsorption of aminotriazoles, like that of the unsubstituted compound itself, can be accompanied by 
formation of surface complexes with copper cations on the electrode with an increase in concentration.

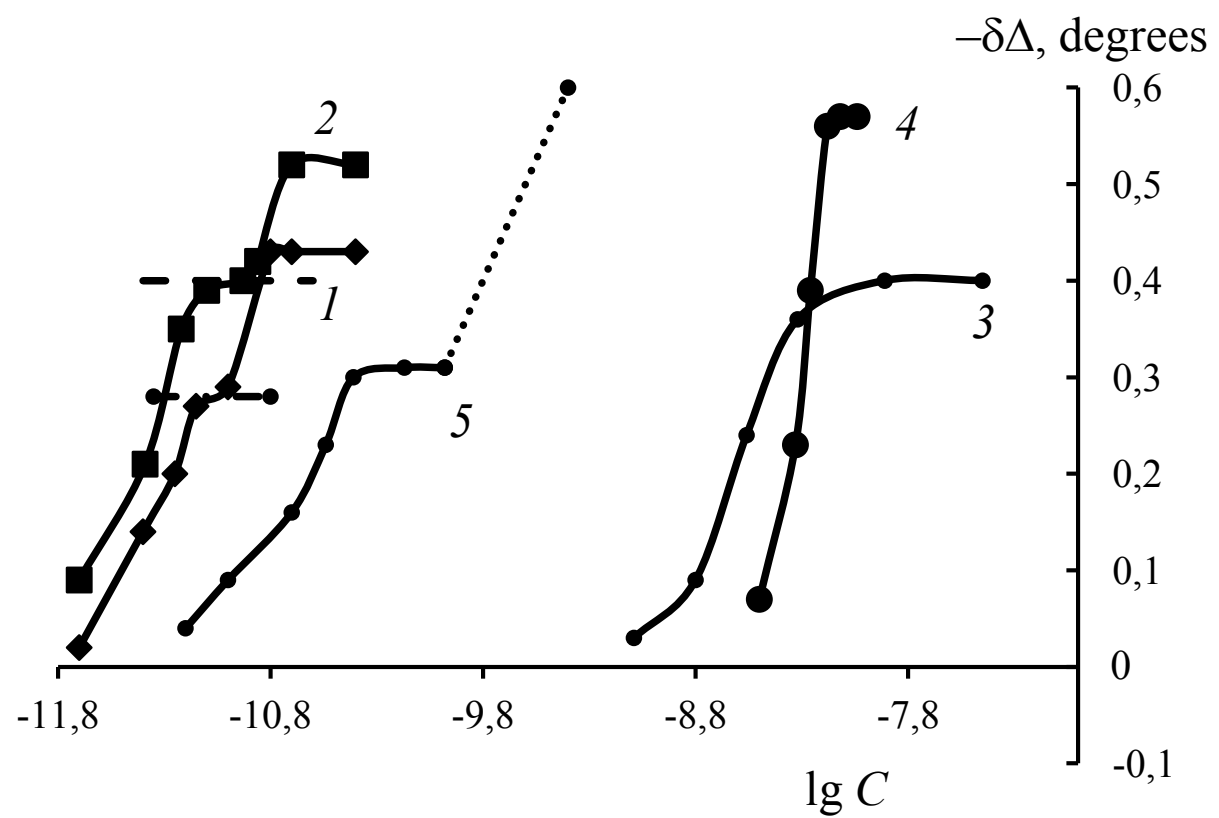

Figure 1. Plots of ellipsometric angle $\Delta$ versus the concentration of triazoles: 1- $H-1,2,4-$ triazole (1), MP-aminotriazole (2), Pentylaminotriazole (3), 3-aminotriazole (4) and 4aminotriazole (5) on copper surface at $E=0.0 \mathrm{~V}$. The dashed line across curves 1 and 2 denotes the regions of nonstationary values of $\Delta$.

On the other hand, incorporation of substituents $\mathrm{R}=\mathrm{C}_{5} \mathrm{H}_{11}$ or $\mathrm{SC}_{5} \mathrm{H}_{11}$ to the structure of 3-AT should increase its adsorption ability, because it increases the hydrophobicity of the compounds. Thus their association with solvent molecules weakens, the surface activity increases, and hence, the adsorption of organic anions on the oxidized copper also increases (Fig. 1). Indeed, as can be seen from the data presented in Table 1, MP-3-AT is a more hydrophobic and hence more surface-active compound than the compounds discussed above. On the plots of $(-\delta \Delta)$ versus $\lg C$ for pentyl-3-AT and MT-3-AT (Fig. 1), the inflections on the respective curves are recognized as representing the formation of a conventional monolayer. Calculation of adsorption isotherms based on Eq. (2) for pentyl3-AT gives $\left(-\Delta G_{\mathrm{a}}^{0}\right)=69 \mathrm{~kJ} / \mathrm{mol}$ and $a=1.8$. A further increase in the hydrophobicity from pentyl-3-AT to MP-3-AT improves the adsorption of substituted aminotriazoles. Calculation of the adsorption isotherms based on Eq. (2) for MP-3-AT gives $\left(-\Delta G_{\mathrm{a}}^{0}\right)=$ $70 \mathrm{~kJ} / \mathrm{mol}$ and $a=1.9$ (Table 1 ).

However, it is worthy of note that no linear correlation is observed between the values of $\log D$ and $\left(-\Delta G_{\mathrm{a}}^{0}\right)$ for the triazole derivatives studied: an increase in the compound hydrophobicity has a much stronger effect on its adsorptivity on transition from 4- and 
especially 3-AT to 1-H-1,2,4-triazole than from pentyl-3-AT to MT-3-AT. Therefore, the hydrophobicity of the studied compounds is an important, but not the only factor that determines the adsorption and inhibiting properties of triazoles. Unfortunately, the small set of substituted 1-H-1,2,4-triazoles does not allow for at least two-factor analysis of the relationship between their chemical structure and the properties studied. One can assume, however, that in addition to the acidic properties, an essential role is also played by the steric factor. In fact, although bulky substituents in the triazole molecule increase the surface activity of the compound, they can hinder the formation of complex compounds it forms with copper.

Comparison of the attraction characteristics of triazoles shows that interaction of particles of substituted 3-AT and 4-AT with the surface and between each other in the adsorption layer is stronger than that for unsubstituted inhibitors, i.e., 1- $H-1,2,4$-triazole and 3-AT.

It is interesting to compare the results of adsorption and electrochemical studies. Although electrochemical measurements were carried out not in pure borate buffer but in the presence of a depassivator $(0.01 \mathrm{~mol} / \mathrm{l} \mathrm{NaCl})$, they provided an opportunity to obtain valuable information about the passivating action of triazoles and their ability to stabilize the passive state of copper under conditions of competitive adsorption of an organic inhibitor and chloride.

Polarization curves for copper in solutions containing various concentrations of $1-\mathrm{H}$ 1,2,4-triazole are presented in Fig. 2. At $C_{\text {in }}>0.11 \mathrm{mmol} / 1$, passivation of copper occurs spontaneously, while at lower $C_{\text {in }}$ the anodic curves contain a region of active dissolution potentials of copper. At $C_{\mathrm{in}}=1.5-10.0 \mathrm{mmol} / 1, E_{\mathrm{pt}}$ changes very slightly, and only addition of $12.5 \mathrm{mmol} / 1$ shifts it to almost $1 \mathrm{~V}$. For this inhibitor, as well as for all other triazoles, a shift of $E_{\text {cor }}$ to more negative values is characteristic, which is usually associated with inhibition of the cathodic reaction similarly to our observation for BTA [21, 22]. Indeed, cathodic polarization curves of copper with all the compounds studied show that they inhibit the depolarizer reduction.

Previously, we have shown [21] that spontaneous passivation of copper in borate buffer containing $\mathrm{Cl}^{-}$anions upon addition of 3 -AT occurs at $C_{\mathrm{in}} \sim 1.5 \mathrm{mmol} / \mathrm{l}$, while $E_{\mathrm{pt}}$ reaches $0.78 \mathrm{~V}$. The stability of the passive state of copper in this solution increases with the concentration of $3-\mathrm{AT}$ and at $C_{\mathrm{in}} \geq 10.0 \mathrm{mmol} / 1 E_{\mathrm{pt}}$ reaches the potential of oxygen evolution, $E_{\mathrm{O}_{2}}$.

Substituted 3-ATs, namely, pentyl-3-AT and MP-3-AT operate at much lower concentrations than 3-AT, and are inhibitors of mixed type (Figs. 3 and 4). Pentyl-AT even at $C_{\text {in }}=0.001 \mathrm{mmol} / 1$ decreases the active dissolution current 15 -fold as compared with the background curve (Fig. 3). In the range of $C_{\text {in }}=0.005-0.5 \mathrm{mmol} / 1$, copper passivation occurs spontaneously without changing $E_{\mathrm{pt}}$. An increase in $C_{\mathrm{in}}$ to $10 \mathrm{mmol} / \mathrm{l}$, shifts $E_{\mathrm{pt}}$ to $0.96 \mathrm{~V}$. 


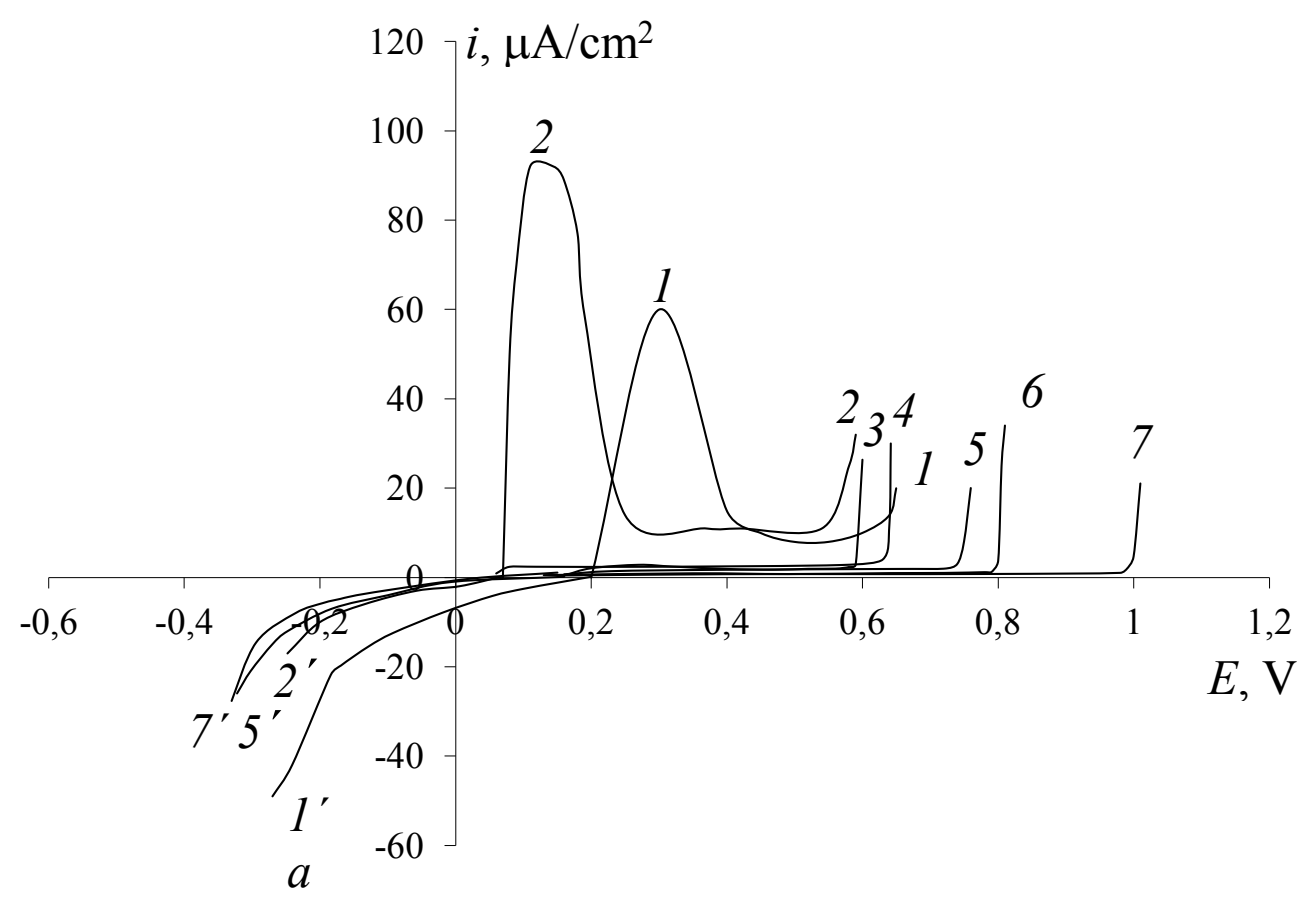

Figure 2. Anodic $(1-7)$ and cathodic $\left(1^{\prime}, 2^{\prime}, 5^{\prime}, 7^{\prime}\right)$ polarization curves for copper in borate buffer solution $\left(\mathrm{pH} \mathrm{7.4)} \mathrm{containing} 10 \mathrm{mmol} / \mathrm{l} \mathrm{NaCl}\left(1,1^{\prime}\right)\right.$ and various concentrations of $1-H$ 1,2,4-triazole (mmol/1): 2,2' $-0.06 ; 3^{\prime}-0.11 ; 4-0.23 ; 5,5^{\prime}-0.56 ; 6-1.1 ; 7,7^{\prime}-12.5$.

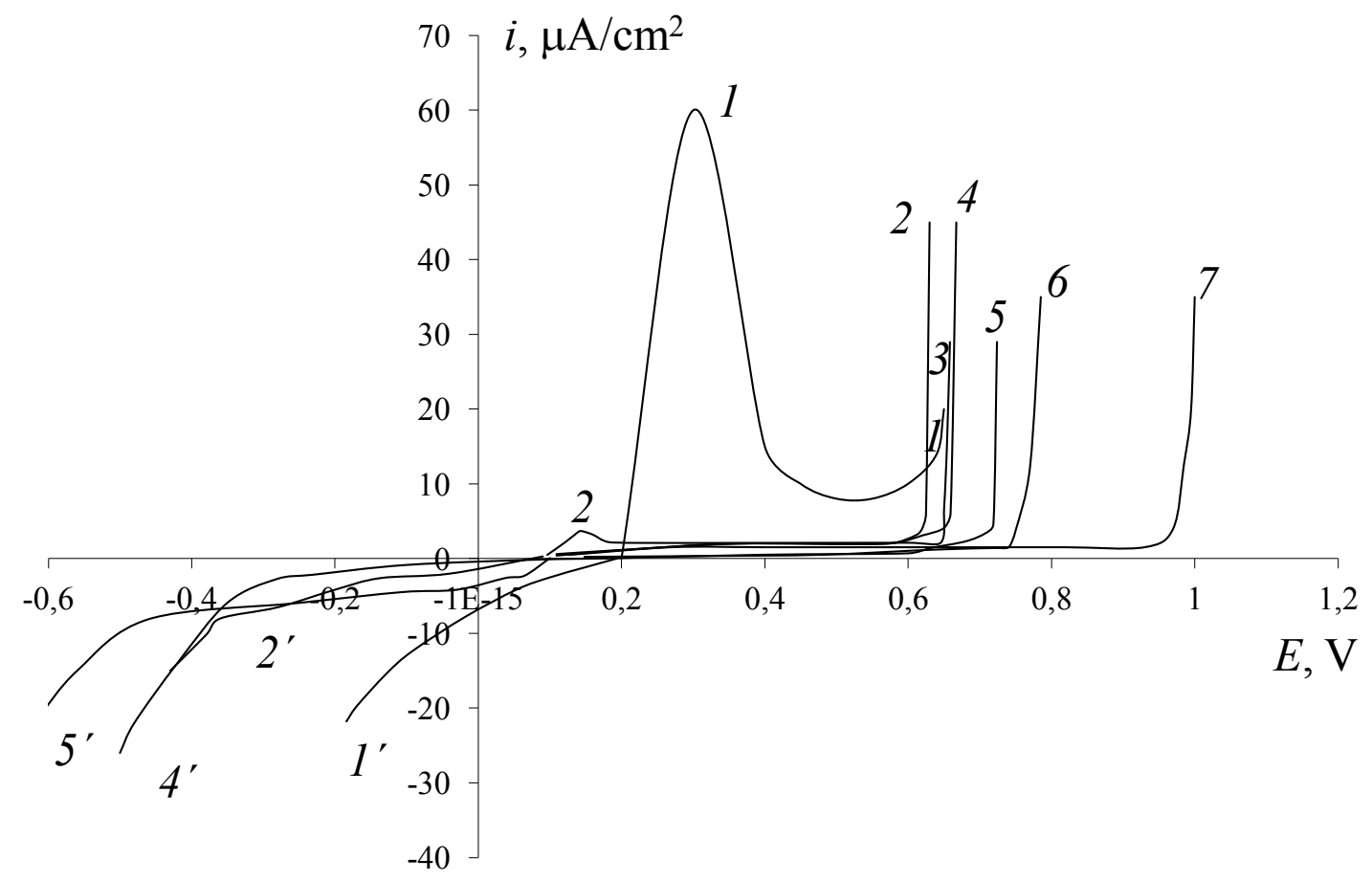

Figure 3. Anodic (1-7) and cathodic $\left(1^{\prime}, 2^{\prime}, 4^{\prime}, 5^{\prime}\right)$ polarization curves for copper in borate buffer solution $(\mathrm{pH} 7.4)$ containing $10 \mathrm{mmol} / 1 \mathrm{NaCl}\left(1,1^{\prime}\right)$ and various concentrations of P-3AT (mmol/l): $2,2^{\prime}-0.001 ; 3-0.005 ; 4,4^{\prime}-0.5 ; 5,5^{\prime}-2.5 ; 6-4.5 ; 7-10.0$. 


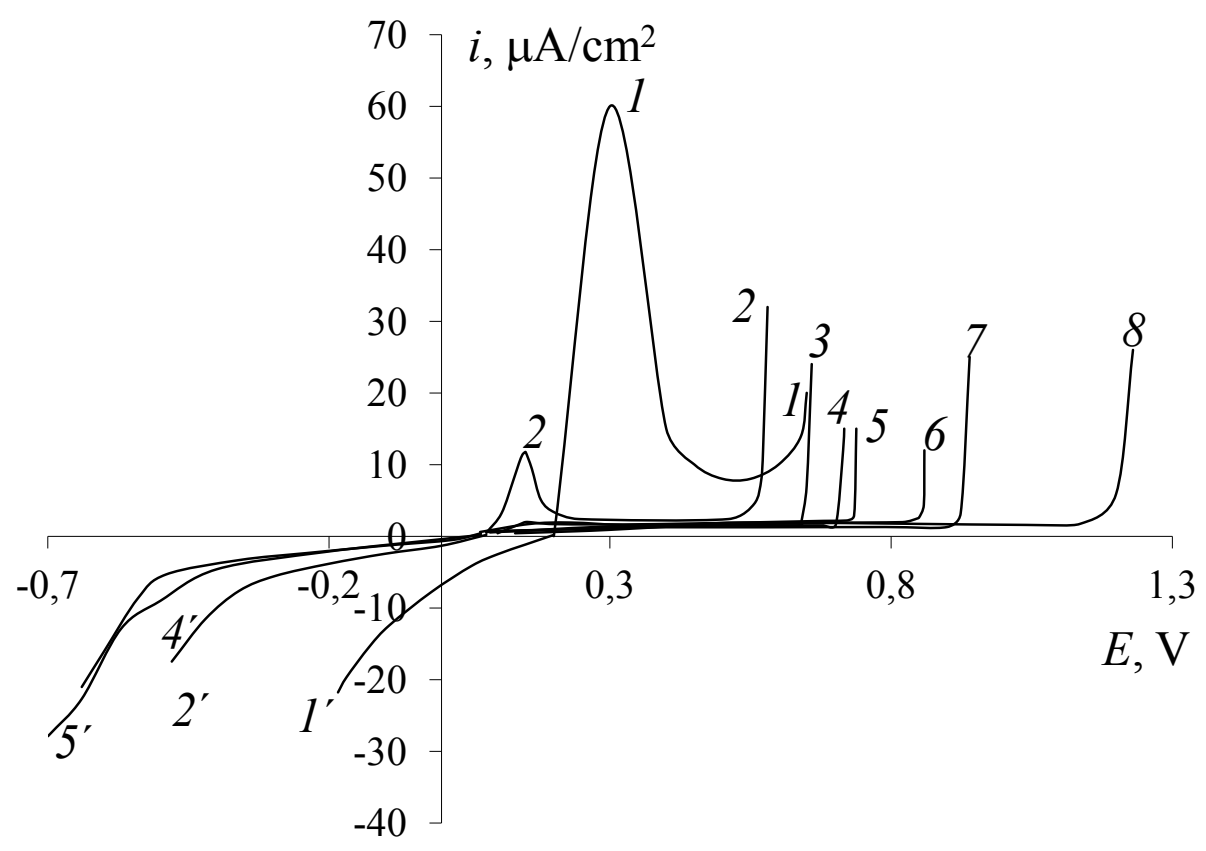

Figure 4. Anodic $(1-8)$ and cathodic $\left(1^{\prime}, 2,^{\prime} 4^{\prime}, 5^{\prime}\right)$ polarization curves for copper in borate buffer solution ( $\mathrm{pH} 7.4)$ containing $10 \mathrm{mmol} / 1 \mathrm{NaCl}\left(1,1^{\prime}\right)$ and various concentrations of MP3-AT (mmol/1): $2,2^{\prime}-0.001 ; 3-0.005 ; 4,4^{\prime}-0.01 ; 5,5^{\prime}-1.5 ; 6-2.5 ; 7-4.5 ; 8-10.0$.

MP-3-AT at $C_{\mathrm{in}}=0.001 \mathrm{mmol} / 1$ also drastically reduces the active dissolution current of copper compared with the background curve (Fig. 4), and even at $C_{\text {in }}=0.005 \mathrm{mmol} / 1$ copper passivation occurs spontaneously without changing the $E_{\mathrm{pt}}$. An increase in $C_{\text {in }}$ to $10 \mathrm{mmol} / 1$ shifted $E_{\mathrm{pt}}$ to $1.2 \mathrm{~V}$, i.e., the passive potential region expands to a greater extent than in the presence of other inhibitors.

Polarization curves for 4-AT differ from similar curves for all the triazoles studied [21]. We failed to achieve spontaneous copper passivation in 4-AT solutions (Fig. 5). When 4-AT concentration in the solution increases, the active dissolution current of copper electrode changes non-monotonically: they decrease at $C_{\text {in }}=0.02-0.25 \mathrm{mmol} / 1$, increase at $C_{\text {in }}=0.25-1.5 \mathrm{mmol} / 1$, and decrease again at $C_{\text {in }}=1.5-10 \mathrm{mmol} / 1$. However, $E_{\mathrm{pt}}$ always regularly increases with an increase in $C_{\text {in }}$. Therefore, 4-AT inhibits the anodic dissolution of copper in general, although its efficiency depends on the potential region of the electrode. In contrast, $E_{\text {cor }}$ decreases, indicating that 4-AT inhibits the cathodic reaction on copper.

Analysis of the results of adsorption and polarization measurements reveals the important role of the anion hydrophobicity (Table 1) and ability to passivate copper. The relatively hydrophilic 3-AT is able to passivate copper in borate-chloride solutions, though a high $C_{\text {in }}$ is required, while a more hydrophilic 4-AT fails to provide spontaneous passivation of copper at all the $C_{\text {in }}$ studied, although the passivation current density of copper decreases in the presence of this triazole. Apparently, the negative effect on the protective properties of 4-AT is also caused by the absence of acidic properties because the 
inhibitor cannot interact with copper surface as an anion. For other triazoles, despite their low acidity, it is impossible to rule out that dissociation of the molecules on the electrode surface increases and they are adsorbed in anionic form.

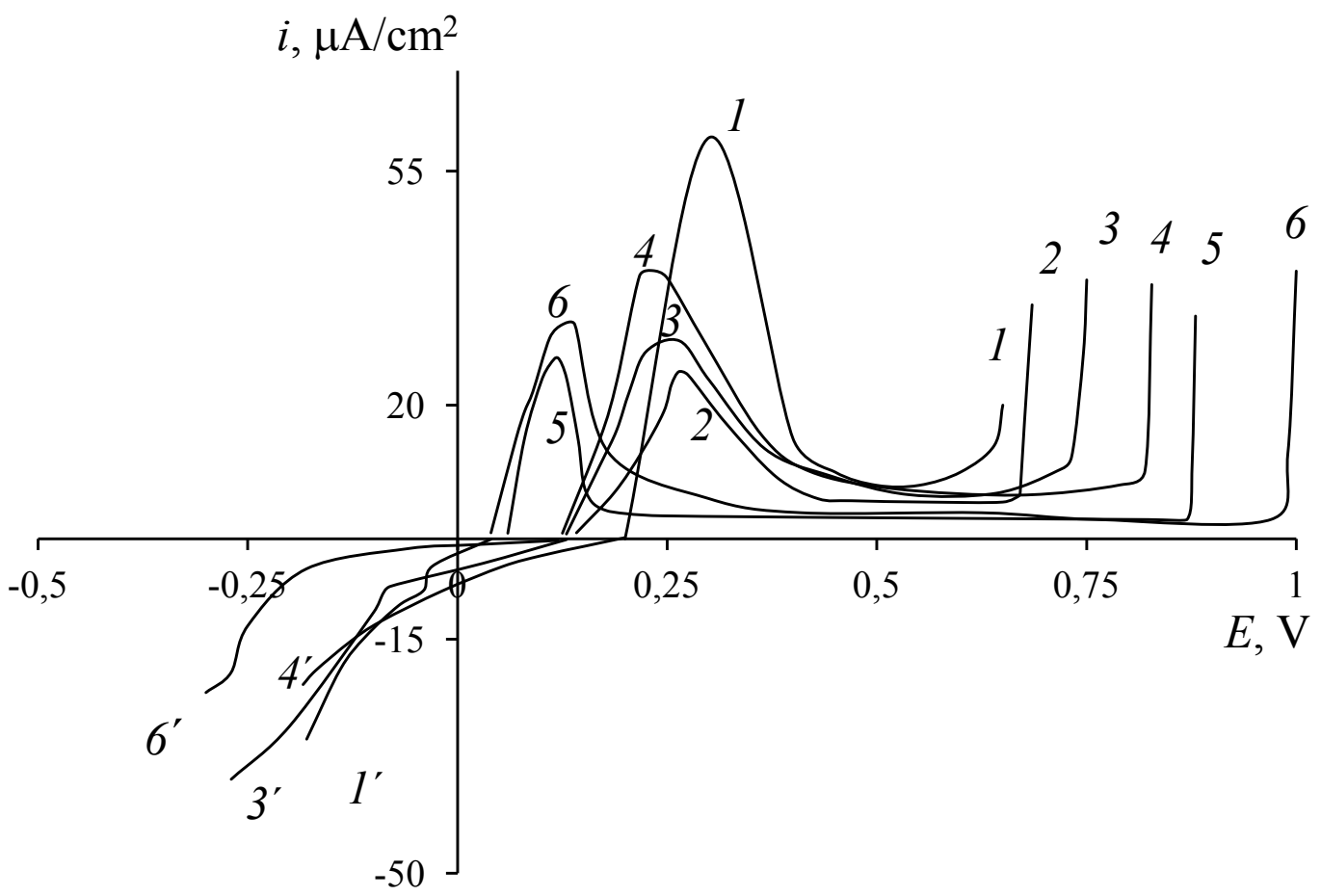

Figure 5. Anodic (1-6) and cathodic $\left(1^{\prime}, 3^{\prime}, 4^{\prime}, 6^{\prime}\right)$ polarization curves for copper in borate buffer solution ( $\mathrm{pH} 7.4)$ containing $10 \mathrm{mmol} / 1 \mathrm{NaCl}\left(1,1^{\prime}\right)$ and various concentrations of 4-AT (mmol/l): $2-0.02 ; 3,3^{\prime}-0.25 ; 4,4^{\prime}-1.5 ; 5-2.5 ; 6,6^{\prime}-10.0$.

The thickness of triazole monolayers on oxidized copper was determined from the results of ellipsometric measurements in a manner similar to that we reported previously $[21,22]$. The complex refractive index calculated from the $\Delta_{0}$ and $\Psi_{0}$ angles was found to be $N_{\mathrm{Cu}}=0.6-3.2 i$ at $E=-0.60 \mathrm{~V}$. By plotting nomograms in the $\delta \Delta-\delta \psi$ plane based on experimental and theoretical curves for various $N_{\text {oxide }}$ values, the complex refractive index of $\mathrm{Cu}_{2} \mathrm{O}$ was found to be $N_{\text {film }}=2.75-0.05 i$. The oxide thickness is about $1 \mathrm{~nm}$. In Eq. (1) the proportionality factor $\alpha$ is $0.89 \mathrm{~nm} / \mathrm{deg}$ (the best match between the theoretical and experimental curves for the refractive index $N_{3-\mathrm{AT}}=2.0 \pm 0.2$ ). Based on the known $\alpha$ and the corresponding changes in the ellipsometric angles for monolayer surface coverage by inhibitors, the following thicknesses were derived (with an accuracy of $\pm 0.1 \mathrm{~nm}$ ): $d=$ $0.28 \mathrm{~nm}$ for $1-H-1,2,4$-triazole $\left(-\delta \Delta=0.31^{\circ}\right), d=0.36 \mathrm{~nm}$ for MP-3-AT $\left(-\delta \Delta=0.40^{\circ}\right)$, and $d=0.25 \mathrm{~nm}$ for pentyl-3-AT $\left(-\delta \Delta=0.28^{\circ}\right)$.

The thicknesses $d=0.36 \mathrm{~nm}$ for 3-AT $\left(-\delta \Delta=0.4^{\circ}\right)$ and $d=0.5 \mathrm{~nm}$ for 4-AT $(-\delta \Delta=$ $\left.0.56^{\circ}\right)$ were determined in [21]. The sizes of the molecules of these inhibitors are $0.22 \mathrm{~nm}$ for 1-H-1,2,4-triazole, $1.24 \mathrm{~nm}$ for 3-pentyl-AT, and $1.38 \mathrm{~nm}$ for the MP-3-AT. 
Thus, based on these calculations, we can assume that molecules of substituted ATs on a copper surface are arranged in a nearly horizontal (flat) position, similarly to unsubstituted 3-AT molecules. It is only 4-AT molecules [21] and anions of 1- $H-1,2,4-$ triazole that are arranged vertically.

\section{Conclusions}

1. A relationship has been established between the adsorption and protective properties of aminotriazoles and their hydrophobicity. Adsorption of all the triazoles studied is polymolecular. However, the first monolayer adjacent to the metal is adequately described by the Frumkin equation on oxidized copper surface at $E=0.0 \mathrm{~V}$. The free energy of adsorption for $1-H-1,2,4$-triazole $\left(-\Delta G_{\mathrm{a}}^{0}\right)$ equals $64 \mathrm{~kJ} / \mathrm{mol}$. From polarization measurements it can be concluded on mixed-type inhibition of copper dissolution by this triazole in neutral chloride solution.

2. Adsorption of even more hydrophilic 3- and 4-aminotriazoles shows lower values of $\left(-\Delta G_{\mathrm{a}}^{0}\right)$ than $1-H-1,2,4-$ triazole, namely, $51-54 \mathrm{~kJ} / \mathrm{mol}$. These compounds are weaker inhibitors than 1-H-1,2,4-triazole.

3. Pentyl- and mercaptopentylaminotriazoles are adsorbed at lower concentrations than all the other triazoles due to their hydrophobicity. The adsorption energy of these compounds is $\left(-\Delta G_{\mathrm{a}}^{0}\right)=69-70 \mathrm{~kJ} / \mathrm{mol}$. They inhibit the anodic dissolution of copper in neutral chloride solution and facilitate passivation at lower concentrations than the $1-\mathrm{H}$ 1,2,4-triazole and its amino derivatives.

4. The possible arrangement of triazole molecules on oxidized copper surface was revealed. Pentyl- and mercaptopentylaminotriazoles are arranged on the surface in a nearly horizontal (flat) position. 4-AT and anions of 1- $\mathrm{H}-1,2,4$-triazole are arranged vertically with respect to the surface.

The $\mathrm{p} K_{\mathrm{a}}$ values and hydrophobicity characteristics were calculated using the ACDLab program available on the Internet at https://ilab.acdlabs.com.

The $\log D$ values were taken for $\mathrm{pH} 7.40$.

This study was supported by the Ministry of Education and Science of the Russian Federation (contract No. 02.G25.31.0007).

\section{References}

1. J. B. Cotton, US Pat. 933979, published 14.08.1963.

2. J. K. Aiken, Products Finishing, 1959, 12, no. 10, 90.

3. I. Dugdale and J. B. Cotton, Corros. Sci., 1963, 2, no. 1, 69.

4. G. W. Poling, Corros. Sci., 1970, 10, 359.

5. Yu. I. Kuznetsov, and L. P. Kazanski, Usp. Khim., 2008, 77, no. 3, 227.

6. K. Cho, J. Kishimoto, T. Hashizume, H. W. Pickering and T. Sakurai, Appl. Surf. Sci., 1995, 87/88, 380. 
7. T. Kosec, D. K. Merl and I. Milošev, Corros. Sci., 2008, 50, 1987.

8. S. Liu, N. Xu, J. Zeng, Z. Feng and R. Xiao, Corros. Sci., 2009, 51, 1356.

9. D. Gallant, M. Pézolet and S. Simard, Electrochim. Acta, 2007, 52, 4927.

10. K. Es-Salah, M. Keddam, K. Rahmouni, A. Srhiri and H. Takenouti, Electrochim. Acta, 2004, 49, no. 17-18, 2771.

11. L. V. Frolova, Yu. I. Kuznetsov and O. O. Zel, Korroz.: Mater. Zashch., 2008, no. 11, 23 (in Russian).

12. Ya. G. Avdeev, L. V. Frolova, Yu. I. Kuznetsov and O. O. Zel, Korroz.: Mater. Zashch., 2010, no. 8, 19 (in Russian).

13. Ya. G. Avdeev, Yu. I. Kuznetsov and O. O. Zel, Korroz.: Mater. Zashch., 2010, no. 7, 12 (in Russian).

14. K. F. Khaled and M. A. Amin, Corros. Sci., 2009, 51, 2098.

15. V. F. Voloshin, O. P. Golosova and L. A. Mazalevskaya, Protection of Metals, 1986, 22, 472.

16. A. N. Onal and A. A. Aksut, Anti-Corrosion Methods and Materials, 2000, 47, no. 6, 339.

17. Yu. V. Karyakin and I. I. Angelov, Chistye khimicheskie reaktivy (Pure chemical reagents), Moscow, Khimiya, 1974, 164, 269 (in Russian).

18. N. P. Andreeva and Yu. I. Kuznetsov, Protection of Metals, 1989, 25, no. 2, 214.

19. Chemical encyclopedia, Vol. 4, Bol'shaya Sovetskaya Entsiklopediya Publishing House, Moscow, 1995, pp. 1251-1254 (in Russian).

20. C. Hanch and A. Leo, Substituent Constants for Correlation Analysis in Chemistry and Biology, Wiley-Interscience, New York, 1979.

21. Yu. I. Kuznetsov, M. O. Agafonkina and N. P. Andreeva, Russ. J. Phys. Chem., 2014, no. 4, 708.

22. N. P. Andreeva, M. O. Agafonkina and Yu. I. Kuznetsov, Korroz.: Mater. Zashch., 2010, no. 9, 7 (in Russian).

23. L. P. Kazansky, I. A. Selyaninov and Yu. I. Kuznetsov, Appl. Surf. Sci., 2012, 259, 385.

24. V. Chicharro, A. Zarabdiel, E. Bernmejo and M. Moreno, Talanta, 2003, 59, 37. 